\title{
Video Article \\ Subcloning Plus Insertion (SPI) - A Novel Recombineering Method for the Rapid Construction of Gene Targeting Vectors
}

\author{
Thimma R. Reddy ${ }^{1}$, Emma J. Kelsall ${ }^{1}$, Léna M.S. Fevat ${ }^{2}$, Sarah E. Munson* ${ }^{3}$, Shaun M. Cowley* ${ }^{1}$ \\ ${ }^{1}$ Department of Biochemistry, University of Leicester \\ ${ }^{2}$ Center for Fisheries, Environment and Aquaculture Sciences \\ ${ }^{3}$ ES Cell Facility, Centre for Core Biotechnology Services, University of Leicester \\ * These authors contributed equally
}

Correspondence to: Shaun M. Cowley at smc57@leicester.ac.uk

URL: https://www.jove.com/video/52155

DOI: doi:10.3791/52155

Keywords: Molecular Biology, Issue 95, recombineering, gap-repair, subcloning plus insertion, transgene, knockout, mouse

Date Published: $1 / 8 / 2015$

Citation: Reddy, T.R., Kelsall, E.J., Fevat, L.M., Munson, S.E., Cowley, S.M. Subcloning Plus Insertion (SPI) - A Novel Recombineering Method for the Rapid Construction of Gene Targeting Vectors. J. Vis. Exp. (95), e52155, doi:10.3791/52155 (2015).

\section{Abstract}

Gene targeting refers to the precise modification of a genetic locus using homologous recombination. The generation of novel cell lines and transgenic mouse models using this method necessitates the construction of a 'targeting' vector, which contains homologous DNA sequences to the target gene, and has for many years been a limiting step in the process. Vector construction can be performed in vivo in Escherichia coli cells using homologous recombination mediated by phage recombinases using a technique termed recombineering. Recombineering is the preferred technique to subclone the long homology sequences $(>4 \mathrm{~kb})$ and various targeting elements including selection markers that are required to mediate efficient allelic exchange between a targeting vector and its cognate genomic locus. Typical recombineering protocols follow an iterative scheme of step-wise integration of the targeting elements and require intermediate purification and transformation steps. Here, we present a novel recombineering methodology of vector assembly using a multiplex approach. Plasmid gap repair is performed by the simultaneous capture of genomic sequence from mouse Bacterial Artificial Chromosome libraries and the insertion of dual bacterial and mammalian selection markers. This subcloning plus insertion method is highly efficient and yields a majority of correct recombinants. We present data for the construction of different types of conditional gene knockout, or knock-in, vectors and BAC reporter vectors that have been constructed using this method. SPI vector construction greatly extends the repertoire of the recombineering toolbox and provides a simple, rapid and cost-effective method of constructing these highly complex vectors.

\section{Video Link}

The video component of this article can be found at https://www.jove.com/video/52155/

\section{Introduction}

The development of gene targeting technologies has enabled the construction of cell lines and mouse models to investigate different biological systems ${ }^{1-3}$. A key first stage in the modification of a genomic sequence is the design and construction of a gene targeting vector ${ }^{4}$. Targeting vectors are plasmid constructs that carry the allele of interest containing the desired modifications(s) flanked by a selection marker (e.g., neomycin), and long genomic regions required for efficient homologous recombination in mammalian cells ${ }^{5}$. Precise gene modification is achieved by the introduction of the targeting vector into embryonic stem (ES) cells ${ }^{6}$ or somatic cells ${ }^{7}$, whereby homologous recombination between identical stretches of DNA sequence on the targeting vector and the genomic locus results in the transfer of the intended modification to the genome by gene conversion ${ }^{8}$. Such modified ES cells can be injected into mouse blastocysts to produce offspring (chimaeras) that can then transmit these modified alleles via the germline ${ }^{9}$. An alternate route to produce transgenic mice involves the microinjection of a gene expression vector into single celled mouse zygotes, which leads to the random integration of the vector in the mouse genome ${ }^{10}$. Traditional methods of vector construction have relied on conventional 'cut and paste' cloning using restriction enzymes and DNA ligases to clone the different selection marker and genomic fragments into a vector backbone. However, an inherent limiting factor of traditional cloning is the positioning and choice of restriction sites, especially with longer DNA sequences. This often necessitates multiple subcloning steps and also introduces extraneous DNA sequences into the vector, which often leads to a lower efficiency of gene targeting.

Recombineering (recombinogenic engineering) is a DNA engineering technology that overcomes these limitations by using homologous recombination (HR) mediated by phage recombination proteins in $E$. coli cells ${ }^{11,12}$. Since any region of a homologous sequence can serve as a substrate of recombineering, the constraints of availability of restriction sites are removed. Large DNA sequences can be seamlessly modified directly in vivo, thus also preserving their structural integrity ${ }^{13}$. Recombineering is very efficient with short homologies $(50 \text { bp })^{14}$ and therefore homology arms (HA) can be conveniently incorporated into synthetic oligo sequences. In a typical recombineering experiment, an oligo or a double stranded DNA (dsDNA) fragment containing HA is electroporated into recombineering competent $E$. coli cells containing the target located either on the chromosome or on a plasmid ${ }^{15}$. The recombination potential is conferred by inducible expression of the Red recombination proteins of the phage $\mathrm{e}^{16,17}$ or the RecET proteins of the rac prophage ${ }^{18}$. The Red/RecE exonuclease converts linear dsDNA to a single-stranded 
DNA (ssDNA) intermediate, which is then bound by its partner, Red/RecT, a single-stranded annealing protein (SSAP) ${ }^{19}$. The annealing of a long ssDNA or a short oligo to its complementary target sequence occurs on the lagging strand of the replication fork and leads to the incorporation of the sequence at the target site. Lagging strand ssDNA recombination is the basis of the high efficiency of recombineering and can be described by the 'beta' recombination model ${ }^{20,21}$

A typical recombineering workflow to build a gene targeting vector involves either of the two following routes. One route involves subcloning the desired genomic region from a mouse BAC clone into a plasmid followed by the sequential insertion of LoxP recombination sites, a selection marker ${ }^{22}$ etc., or the alternative route involves targeting the BAC genomic locus with the different targeting vector elements by multiple rounds of recombineering ${ }^{10,23}$ and then subcloning the modified locus into a plasmid by gap repair cloning ${ }^{24,25}$. Variations on this theme have been used in different high-throughput recombineering pipelines as part of large mouse production programs ${ }^{26,27}$. However, these procedures involve complicated and lengthy stages, require the use of specialized vectors and E. coli strains (e.g., Cre expressing cells) and utilize one or more intermediate steps of vector DNA purification and re-transformation (Table 1). Subcloning plus insertion (SPI) is a novel recombineering technique that combines beta recombination and gap repair cloning into a single process (Figure 1). SPI vector assembly is simple, quick and flexible and offers significant improvement on standard recombineering approaches (Table 1). Here, we demonstrate the ease and utility of using SPI for different vector construction applications with a particular emphasis on constructing non-standard and challenging vector designs. Test cases included the construction of a fluorescent reporter knockin vector, a dual tagged protein expression knockin vector, a BAC fluorescent reporter vector and a conditional knockout vector. These examined variously the requirement to localize a cell surface receptor, purify a nuclear protein complex or conditionally ablate the expression of a gene.

\section{Gene Targeting Design}

1. Order an appropriate BAC clone covering the genomic region of interest. Ensure that this is isogenic to the type of ES cells to be modified e.g., RPCl-23 and RPCl-24 BAC clones for gene targeting with $\mathrm{C} 57 \mathrm{BL} / 6 \mathrm{ES}$ cells.

2. Apply conventional gene targeting criteria when designing the targeting vector. Key parameters include whether the modification is required to be constitutive or conditional, defining critical exons (CE) for deletion in a gene knockout strategy and spacing and placement of intronic cassettes.

NOTE: Each of these has been discussed in detail elsewhere ${ }^{10}$.

3. Choose genomic regions each of 5-6 kb flanking the target modification site. NOTE: The size of the subcloned insert is therefore typically $10-12 \mathrm{~kb}$, although the upper limit can be as high as $80 \mathrm{~kb}$ with a low copy subcloning plasmid like p15A, pBR322 etc. and up to $200 \mathrm{~kb}$ with a BAC vector.

\section{Multiplex Recombineering Oligos}

1. Design oligos for the plasmid backbone (subcloning plasmid) and the selectable marker (insertion cassette). Design each oligo such that it contains $180 \mathrm{bp} \mathrm{HA}$ flanking the genomic target site and $20 \mathrm{bp}$ of specific priming sequence of the insertion cassette or the subcloning plasmid (Figure 2).

NOTE: Homology arms should not contain any repetitive elements, presence of repetitive elements will result in incorrect targeting and subcloning. Repetitive elements can be detected by using web based tools such as the "Repeat Masker".

2. Include a unique restriction enzyme (RE) site on one of the vector oligos to linearize the gene targeting vector for ES cell targeting (Figure 2)

3. Check oligo parameters using an oligo analyzer program. Take care to avoid secondary structures in the priming regions.

4. Shorter HA (50 bp) of the insertion cassette is tolerated and yields lower number of recombinants but ensure that the subcloning plasmid HA is always longer (180 bp).

5. Determine the orientation of the genomic DNA insert of the particular BAC clone using web based tools like "cloneDB". Establish the direction of replication from OriS by checking the map of the BAC plasmid backbone used in the BAC library construction.

NOTE: OriS is usually opposite to the transcriptional direction of the Chloramphenicol (Chl) marker for all commonly used BAC plasmids.

6. Add two terminal phosphorothioate (PTO) bonds to the 5' end of the oligo that is opposite to the direction of replication on the BAC clone. Add a 5' phosphate modification to the reverse oligo (Figure 2).

NOTE: The asymmetric phosphorothioated PCR cassette upon Red digestion generates an ssDNA intermediate that can prime the lagging strand of the replication fork.

NOTE: Maximal multiplex recombination frequency is observed with the lagging strand protected cassettes. Leading strand protected or dual protected cassettes may not produce equivalent recombination efficiencies at some loci.

7. Order oligos with PAGE or HPLC purification.

\section{BAC Clone Transformation with pSC101 BADgbaA Recombineering Plasmid.}

1. To make the E. coli strain bearing the BAC recombineering proficient, transform it with the pSC101 BADgbaA plasmid containing the Red genes ${ }^{16}$.

NOTE: The pSC101 BADgbaA plasmid contains the Red and RecA genes under the control of the Arabinose inducible araC- $P_{B A D}$ promoter, a tetracycline selection marker and the temperature sensitive pSC101 replicon.

1. Stab a sterile pipette tip in the BAC agar culture and inoculate $5.0 \mathrm{ml}$ of lysogeny broth (LB) $\mathrm{pH} 8.0$ containing $12.5 \mu \mathrm{g} \mathrm{m}{ }^{-1} \mathrm{Chl}$. Grow at $37^{\circ} \mathrm{C}$ for $5 \mathrm{hr}$ shaking at $200 \mathrm{rpm}$.

2. Chill $10 \%(\mathrm{v} / \mathrm{v})$ glycerol solution, microcentrifuge tubes and electroporation cuvettes on ice. Cool a refrigerated large centrifuge and microcentrifuge to $4{ }^{\circ} \mathrm{C}$.

3. Determine the optical density (OD) of the culture using a spectrophotometer and measure absorbance at $600 \mathrm{~nm}$. Prepare electrocompetent cells (as described below) when an $\mathrm{OD}_{600}$ reading of 0.3 to 0.8 is reached. 
4. Spin down cells in a $50 \mathrm{ml}$ centrifuge tube in a large centrifuge at $1,216 \times \mathrm{g}$ for 5 min at $4{ }^{\circ} \mathrm{C}$.

5. Wash cells with $1 \mathrm{ml}$ of chilled $10 \%$ glycerol and spin down cells at $17,949 \times \mathrm{g}$ for $20 \mathrm{sec}$ at $4{ }^{\circ} \mathrm{C}$. Perform the wash step a total of 3 times.

6. Resuspend the cells in a total volume of $50 \mu \mathrm{l}$ of $10 \%$ glycerol and add $10-200 \mathrm{ng}$ of the pSC101 BADgbaA recombineering plasmid. Obtain a single cell suspension by pipetting up and down several times and then transfer the cells to a pre-chilled $1 \mathrm{~mm}$ gap electroporation cuvette.

7. Electroporate the cells with a setting of $1.8 \mathrm{kv}, 25 \mu \mathrm{F}$ and $200 \Omega$.

NOTE: Check the correct settings for each brand of electroporator. A time constant of electroporation less than 4 indicates the presence of salt and other impurities.

8. Immediately recover the cells in $1 \mathrm{ml}$ of LB and transfer the cells to a $50 \mathrm{ml}$ centrifuge tube.

9. Grow the BAC gbaA culture at $30^{\circ} \mathrm{C}$ for $2 \mathrm{hr}$ shaking at $200 \mathrm{rpm}$.

NOTE: The pSC101 BADgbaA plasmid is lost when cells are grown at $37^{\circ} \mathrm{C}$ due to inactivation of the temperature sensitive RepE replication factor. Grow gbaA cells at $30^{\circ} \mathrm{C}$ to maintain the recombineering functions.

10. Add $9 \mathrm{ml}$ of LB containing $12.5 \mathrm{\mu g} \mathrm{ml}^{-1} \mathrm{Chl}$ and $4 \mathrm{\mu g} \mathrm{ml}^{-1}$ Tetracycline (Tet) to the recovered BAC gbaA culture. Grow the BAC gbaA culture $\mathrm{O} / \mathrm{N}$ at $30^{\circ} \mathrm{C}$ shaking at $200 \mathrm{rpm}$.

NOTE: The transformation efficiency of electroporating the supercoiled gbaA plasmid is sufficiently high to permit saturation growth $\mathrm{O} /$ $\mathrm{N}$ in liquid media.

\section{Preparation of Insertion Cassettes and Subcloning Plasmids}

1. To incorporate HA into the insertion cassette(s) and the subcloning plasmid, perform polymerase chain reaction (PCR) using the long modified oligos as described below.

1. Optional: to prevent plasmid carryover into the recombineering reaction, use an R6K origin or similar narrow host range plasmid template to amplify the insertion cassette.

2. Alternatively, linearize the plasmid template using an RE digest (Table 2). Choose a RE that cuts outside the PCR amplification region and is heat inactivated. Heat inactivate the RE as recommended by the manufacturer.

3. Set up polymerase chain reaction (PCR) using a high-fidelity hotstart DNA polymerase system. Prepare a PCR master mix as detailed (Table 3). Perform thermal cycling as shown (Table 3).

2. Analyze PCR products by agarose gel electrophoresis. Load $1-5 \mu$ l of each $P C R$ onto a $1 \%(w / v)$ agarose gel containing $0.5 \mathrm{mg} \mathrm{m}^{-1} \mathrm{Ethidium}^{-1}$ Bromide (EtBR).

NOTE: The presence of non-specific amplification products does not interfere in the recombineering reaction. In some cases however, primer-dimers may decrease recombineering efficiency.

3. Purify PCR products using a PCR purification kit.

1. Optional: remove the plasmid template from PCRs by treatment with Dpnl followed by PCR clean-up. Elute DNA in a minimal volume of sterile deionized water (as recommended by the manufacturer).

NOTE: Addition of Dpnl to unpurified PCR reactions results in reduced efficiency of cleavage of the methylated plasmid DNA template.

4. Quantify PCR amplified DNA by agarose gel analysis against a known set of DNA standards e.g., $\lambda$ HindIII digest or by using a nanodrop spectrophotometer.

\section{Subcloning Plus Insertion}

1. Dilute the O/N BAC gbaA culture 50 fold by adding $200 \mu \mathrm{l}$ in $10 \mathrm{ml} \mathrm{LB+Chl+Tet.} \mathrm{Grow} \mathrm{at} 30^{\circ} \mathrm{C}$ shaking at $200 \mathrm{rpm}$ for $1 \mathrm{hr} 50 \mathrm{~min}$. Include an sample to be used as negative control.

2. Chill all recombineering materials and equipment to $4^{\circ} \mathrm{C}$ as described in step 3.1.2.

3. Pour LB agar pH 8 plates containing the correct concentration of the appropriate selective antibiotic(s) that will allow selection of the DNA fragment that is inserted as well as for the selection of the subcloning vector (Table 4).

NOTE: Some antibiotics are $\mathrm{pH}$ sensitive. Use LB agar $\mathrm{pH} 8$ as a rule.

4. Prepare a $10 \%(\mathrm{w} / \mathrm{v})$ solution of L-Arabinose. Filter sterilize through a $0.2 \mu \mathrm{m}$ syringe filter. NOTE: Arabinose induces the expression of the recombineering proteins from the gbaA plasmid.

5. Check the $\mathrm{OD}$ of the $\mathrm{BAC}$ gbaA culture using a spectrophotometer. Once an $\mathrm{OD}_{600}$ of $0.25-0.3$ is reached, induce the recombineering proteins as described in the following step.

6. Add $200 \mu \mathrm{l}$ of the $10 \%$ Arabinose solution to $10 \mathrm{ml}$ of the BAC culture to achieve a final concentration of Arabinose of $0.2 \%$. Include an uninduced culture (without Arabinose) to be used as negative control.

7. Transfer the BAC culture to a $37{ }^{\circ} \mathrm{C}$ shaking incubator and induce Red expression for 45 min shaking at $230 \mathrm{rpm}$. NOTE: Expression of Red proteins is inefficient at $30^{\circ} \mathrm{C}$.

8. Spin down cells and wash with $10 \%$ glycerol 3 times as described in step 3.1.5.

9. Add $600-1,000 \mathrm{ng}$ each of the subcloning plasmid and the insertion cassette(s) to the recombineering reaction. Include a vector only and vector plus single insert controls to check the recombination proficiency and integrity of the vector and the cassettes.

10. Obtain a single cell suspension by pipetting up and down. Perform electroporation as described in step 3.1 .7 and subsequent recovery in $1 \mathrm{ml}$ LB at $37{ }^{\circ} \mathrm{C}$ for $1 \mathrm{hr}$ for multi-copy plasmids or in $10 \mathrm{ml}$ of LB at $37^{\circ} \mathrm{C}$ for $3 \mathrm{hr}$ for BAC vectors.

11. Plate different dilutions of the recovered culture e.g., $90 \%, 10 \%, 1 \%$ on the dual selection agar plates and grow at $37{ }^{\circ} \mathrm{C}$ for $16 \mathrm{hr}$. 


\section{Analysis of Recombinants}

1. Optional: Pick 6 to 12 colonies and perform colony PCR using a HA flanking primer and an insert specific primer. Include the subcloning plasmid and the insertion cassette plasmid as well as the parent BAC clone as negative controls.

1. Perform agarose gel analysis of the colony PCRs. Identify positive clones by the presence of a bright band at the expected size. NOTE: The high efficiency of the SPI cloning process generates mostly correct recombinants.

2. Pick colonies into $5 \mathrm{ml} \mathrm{LB} \mathrm{pH} 8$ containing the selective antibiotic and grow $\mathrm{O} / \mathrm{N}$ at $37^{\circ} \mathrm{C}$.

3. Prepare DNA minipreps using a column purification kit as per the manufacturer's instructions.

4. Prepare BAC miniprep DNA using standard phenol-chloroform isolation or a similar protocol.

5. Perform RE digests on the miniprep DNA. Separate DNA by agarose gel electrophoresis. Analyze RE patterns to identify the clones containing the expected fragments sizes of the correct targeting vector. Choose an RE that clearly discriminates between the vector lacking insert(s) and the vector containing the insert(s).

6. Optional: to obtain cells that are devoid of the BAC, which is still present in the $E$. coli after recombineering, use the miniprep plasmid DNA to transform DH5alpha or DH10B E. coli cells.

7. Perform DNA sequencing across the HA and insertion cassette to check oligo synthesis errors.

\section{Representative Results}

\section{Knockin Vectors}

Knockin targeting vectors reflect the need to introduce a novel sequence feature in the genome including single base pair substitution in a protein coding region, the fusion of a fluorescent marker or an affinity tag to a protein or the integration of a gene expression cassette. To test the application of SPI in knockin vector construction strategies, two different test cases were examined. Dnttip1 encodes the deoxynucleotidyltransferase, terminal, interacting protein 1A (TDIF1) that together with class I histone deacetylase (HDAC) form a mitotic deacetylase complex (MiDAC) ${ }^{28}$. To investigate the role of Dnttip1 in cell division, a tandem-affinity tagging approach was taken to isolate the TDIF1 interacting proteins. Previous attempts to subclone a portion of the Dnttip1 gene using a p15A vector containing long homology regions resulted in low gap repair efficiency and frequent aberrant recombination products (data not shown). Thus, the construction of a knockin vector at the Dnttip1 locus provided a challenging recombineering exercise. A SPI strategy was designed to subclone a $12 \mathrm{~kb}$ section of the Dnttip1 gene spanning the last exon (exon 13) into a low copy $\mathrm{p} 15 \mathrm{~A}$ vector and to simultaneously insert a dual affinity tag selection cassette into exon 13, replacing the stop codon (Figure 3A). The 2X FLAG-calmodulin binding protein (CBP) linked FRT-PGK-em7-Neomycin (Neo)-BGhpA-FRT cassette $(2.0 \mathrm{~kb})$ was amplified from an RE linearized plasmid using dual PTO modified oligos that contained 120 bp HA flanking the Dnttip 1 stop codon. A p15A zeoDnttip1 subcloning vector $(1.7 \mathrm{~kb})$ was constructed that contained $200 \mathrm{bp}$ regions homologous to the ends of the Dnttip1 sequence to be subcloned. The Dnttip1 subcloning plasmid was RE linearized and PCR amplified using 20 bp modified oligos that generated a leading strand protected vector. The PCR products were Dpnl treated, purified and co-electroporated into recombineering competent Dnttip1 BAC E. coli cells as well as uninduced control cells. The SPI reactions were plated on Zeocin (Zeo) and Kanamycin (Kan) containing agar plates (Figure 3B). SPI produced the correctly modified Dnttip1 knockin vector in all of the 12 recombinants analysed (Figure 3C). DNA sequencing verified the absence of any errors resulting from oligo synthesis or PCR amplification.

Another example of SPI involved the in-frame insertion of an enhanced yellow fluorescent protein (eYFP) linked selection cassette at the $P 2 r x 1$ gene. The $P 2 r x 1$ gene encodes a G-protein-coupled receptor that functions as an ATP-gated ion channe ${ }^{29}$. Linkage to YFP fluorescence permits the tracking of the $\mathrm{P} 2 \times 1$ receptor on the cell surface in various functional assays. $P 2 r \times 1$ is another difficult locus that has presented significant problems with conventional recombineering methodologies (data not shown). Using SPI, a $12 \mathrm{~kb}$ segment of the $P 2 r x 1$ gene encompassing the terminal exon (exon 12) was subcloned into a p15A zeo vector and modified with the concerted insertion of an eYFP-LoxP flanked Neo cassette replacing the stop codon in exon 12 to construct the $P 2 r \times 1$-eYFP knockin vector (Figure 3D). In this example, the lagging strand protected p15A vector (1.7 kb) contained $230 \mathrm{bp} \mathrm{HA}$ and the eYFP cassette contained $50 \mathrm{bp}$ HA and was also left unmodified. The eYFP insertion cassette (2.7 $\mathrm{kb})$ was assembled by splicing overlap PCR of the eYFP gene, PCR amplified from pEYFP-C1, and the LoxP- PGK-em7-Neo-BGhpA-LoxP cassette, PCR amplified from pL452 (NCI, Frederick). The SPI reaction produced hundreds of colonies (data not shown). RE analysis of DNA minipreps prepared from 11 clones showed the majority contained the correctly assembled P2rx1-eYFP knockin vector (Figure 3E). Additional validation by DNA sequencing showed error free insertion of the eYFP cassette. However, some of the clones showed profiles of the untagged gap repaired and the tagged gap repaired plasmids in the same cell (lanes 6-9). A few samples also contained incorrectly gap repaired (lane 2 ) or mistargeted plasmids (lanes 4 and 5). The failure of concurrent subcloning and targeting in some SPI recombinants highlights the limits of efficient SPI cloning when using large cassettes $\left(>3 \mathrm{~kb}\right.$ ), which arise from the constraints on Red processivity of long DNA fragments ${ }^{30}$, or if using short HA. Indeed, increasing the HA of the eYFP cassette to 200 bp increased SPI efficiency and the correct targeting of the cassette (data not shown). Gene targeting with the P2rx1-eYFP knockin vector in JM8.N4 mouse ES cells (C57BL/6 strain) generated 6 positive clones out of 96, which contained the correctly targeted $P 2 r \times 1-e Y F P$ sequence (Figure 3F).

\section{BAC Reporter Vectors}

A BAC clone of the target gene often contains all the requisite upstream and downstream regulatory elements e.g., enhancers, UTRs etc. as well the endogenous promoter to drive gene expression at natural levels ${ }^{31}$. A BAC reporter vector is therefore the preferred vehicle to recapitulate the endogenous expression pattern of a gene ${ }^{32}$. However, the large size of a BAC plasmid (up to $200 \mathrm{~kb}$ ) presents significant practical problems with transfecting an intact BAC into cells ${ }^{33}$. Reducing the size of the BAC genomic insert through BAC trimming ${ }^{34,35}$, while retaining the necessary regulatory elements of a gene, enables easier handling of the $B A C$ and results in a more efficient transfection. Current $B A C$ engineering technology involves multiple rounds of recombineering to achieve this goal ${ }^{35}$. To demonstrate the utility of SPI in BAC trimming, a pBeloBAC11 $\mathrm{BAC}$ vector was used to subclone a $30 \mathrm{~kb}$ genomic sequence including the full length $P 2 r \times 1$ gene from the $168 \mathrm{~kb} P 2 r \times 1 \mathrm{BAC}$ together with the simultaneous insertion of an eYFP cassette in the P2rx1 gene (Figure 4A). The pBeloBAC11 zeo vector backbone (6.5 kb) containing 180 bp HA was PCR amplified with modified oligos that generated a lagging strand protected vector. The same eYFP Neo cassette (3 kb), used in the previous $P 2 r \times 1$ knockin vector construction, was PCR amplified using lagging strand protected oligos containing 180 bp HA and targeted 
the $P 2 r x 1$ exon 12 replacing the stop codon. Following combined electroporation of the eYFP cassette and the pBeloBAC11 zeo vector into $P 2 r x 1$ BAC cells expressing the gbaA recombineering proteins, the culture was recovered in $10 \mathrm{ml} \mathrm{LB} \mathrm{pH} 8$ for $3 \mathrm{hr}$ at $37 \mathrm{C}$ to segregate the BAC plasmids. Pure recombinants were selected on Zeo and Kan agar plates and were observed at a frequency of $2 \times 10^{-6}$. Colony PCR genotyping analysis revealed successful BAC trimming in the 3 out of 6 clones that were analysed (Figure 4B). Long range PCR amplification across the eYFP insertion site confirmed the correct cassette incorporation in the three positive clones (Figure 4B). The three clones lacking the $e Y F P$ insert were also incorrectly gap repaired at the 5' end, though the cause of mistargeting of the eYFP cassette in these clones may be separate to the correct closure of the 5' BAC end. The three eYFP positive BAC clones were further analysed with RE digests and showed the expected pattern of the correctly trimmed eYFP recombinant BAC (Figure 4C). Sequence analysis revealed absence of errors in the eYFP cassette in only 1 clone out of the 3 positives.

\section{Conditional Knockout (cko) Vectors}

Conditional ablation of gene expression is an important tool to investigate developmental processes or to study biological systems at a particular time point. A conditional gene knockout strategy typically involves the placement of LoxP recombination sites surrounding a critical exon (CE). The deletion of a CE upon Cre expression or activation produces a frameshift and a premature stop codon, resulting in degradation of the mRNA due to nonsense mediated decay (NMD). The construction of a conditional gene targeting vector is a complex task and involves several steps of subcloning, targeting and transformation ${ }^{22}$. SPI offers a convenient route to simplify this process. As a test case, a conditional allele of the Zrsr2 gene was constructed using the SPI methodology (Figure 5A). The Zrsr2 gene encodes a splicing factor and a single copy is located on the $\mathrm{X}$ chromosome. The conditional status of gene deletion is particularly important in this instance to control for the possibility of cells adapting to the lack of Zrsr2 during ES cell selection in a constitutive gene deletion targeting strategy. SPI was performed to subclone a $10 \mathrm{~kb}$ portion of the ZrSr2 gene with concurrent insertion of two different LoxP flanked selection cassettes. The FRT-PGK-em7-Neo-FRT-LoxP cassette (2 $\mathrm{kb})$ was PCR amplified from RE digested pL451 using lagging strand protected oligos that contained 180 bp HA targeting the ZrSr2 intron 2. A second cassette containing Rox-PGK-em7-Blasticidin (Bsd)-Rox-LoxP (2 kb) was PCR amplified from an R6K plasmid with lagging strand protected oligos containing $180 \mathrm{bp} \mathrm{HA}$ identical to a downstream region in intron 3 . The two LoxP sites flanked exon 3 , the CE whose deletion upon conditional Cre activation results in a frameshift and introduces a premature stop codon. The Zrsr2 subcloning plasmid (1.6 kb) was PCR amplified from an RE linearised p15A zeo plasmid using lagging strand protected oligos containing $180 \mathrm{bp} H \mathrm{~A}$ matching the ends of the $10 \mathrm{~kb}$ Zrsr2 sequence. SPI reactions were performed as described before and plated on Zeo+Neo+Bsd plates. The ZrSr2 conditional targeting vector was successfully assembled in most of the 12 recombinants examined (Figure 5B). Further DNA sequencing analysis showed correct insertion of both the selection markers in the ZrSr2 cko vector.

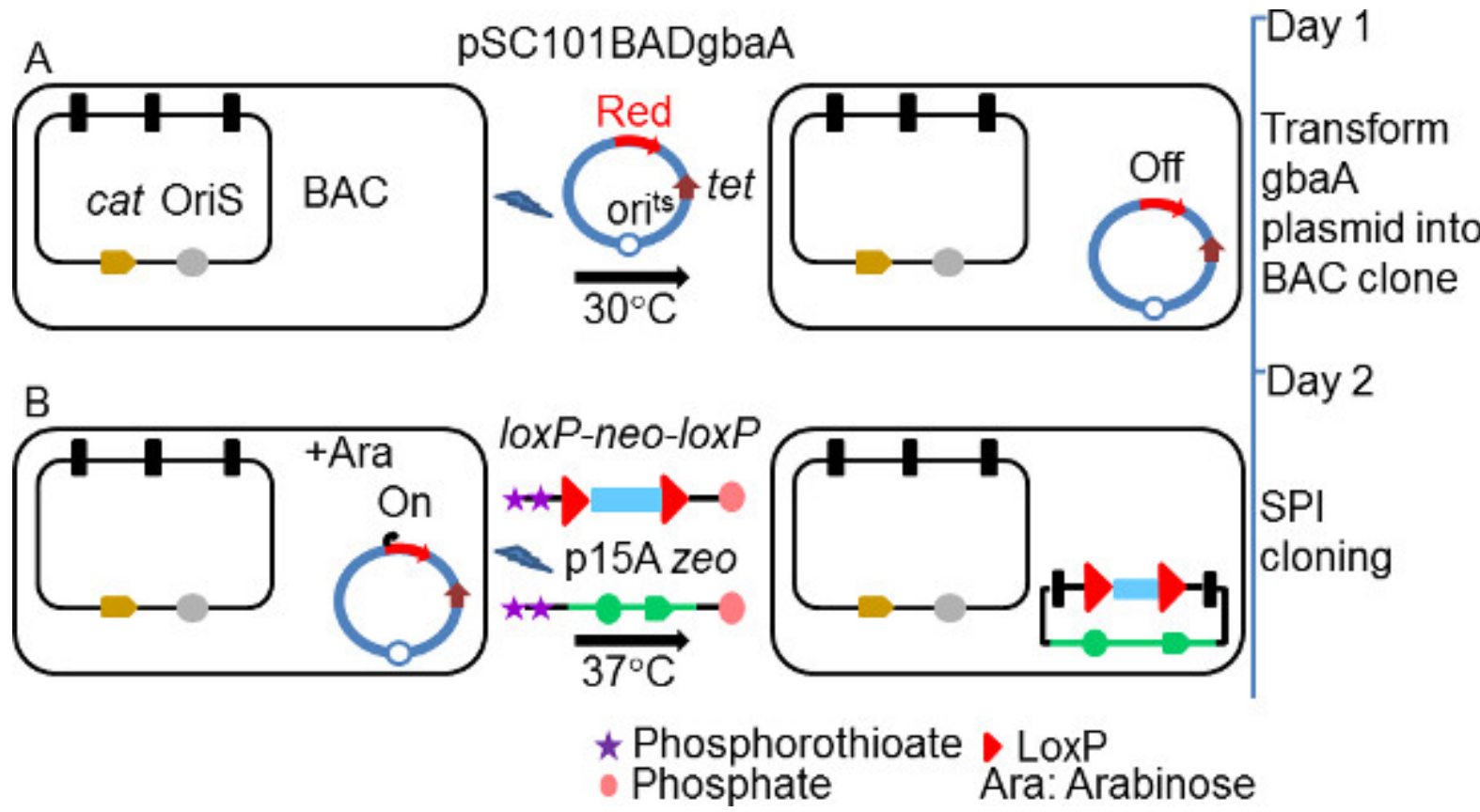

Figure 1. SPI cloning. Overview of the SPI cloning process combining cassette insertion and subcloning in a single step. (A) The BAC clone is transformed with the pSC101 BADgbaA plasmid and grown at 30C. (B) Following arabinose induction to express the Red proteins, the asymmetric modified insertion cassette and subcloning plasmid are introduced into the BAC clone and selected with both the insertion and subcloning markers to generate the final vector. Please click here to view a larger version of this figure. 


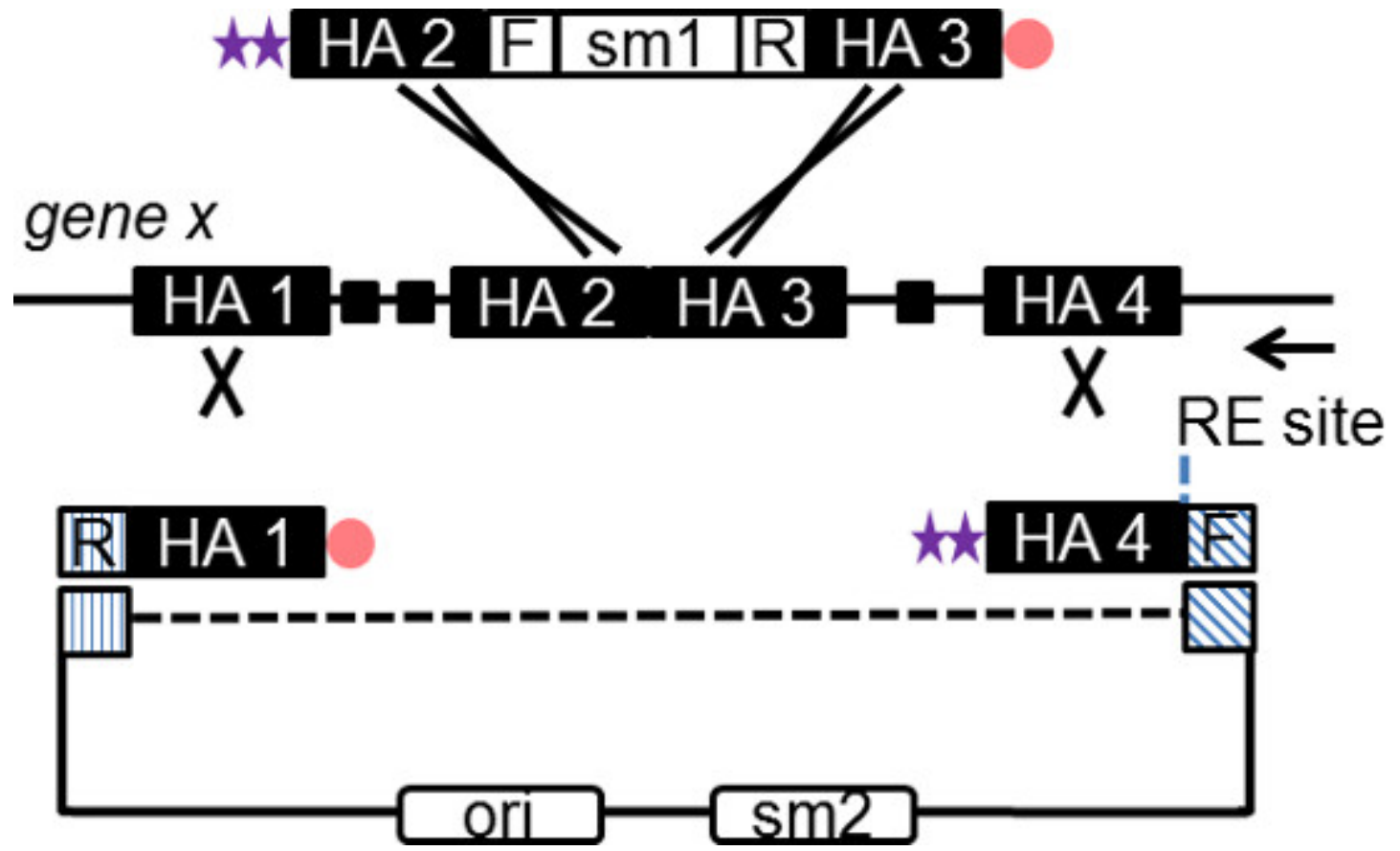

Figure 2. Schematic illustrating the design of SPI recombineering oligos. The subcloning plasmid and insertion cassette are both generated by PCR using a combination of terminal PTO and phosphate modified oligos. The PCR fragment upon Red digestion in vivo, produces an ssDNA intermediate, which anneals to the lagging strand of the replication fork. Gene specific HA of 50-180 bp is incorporated into each oligo as shown. The arrow indicates the direction of DNA replication across a candidate gene. Dashed line represents subcloning plasmid sequence not incorporated into the PCR product. RE site, restriction enzyme site of linearization of final vector; F, forward sequence (20 bp) specific to the subcloning plasmid or insertion cassette; $\mathrm{R}$, reverse complement sequence (20 bp) of the plasmid or cassette; sm, selection marker. Please click here to view a larger version of this figure. 
A

Dnttip1

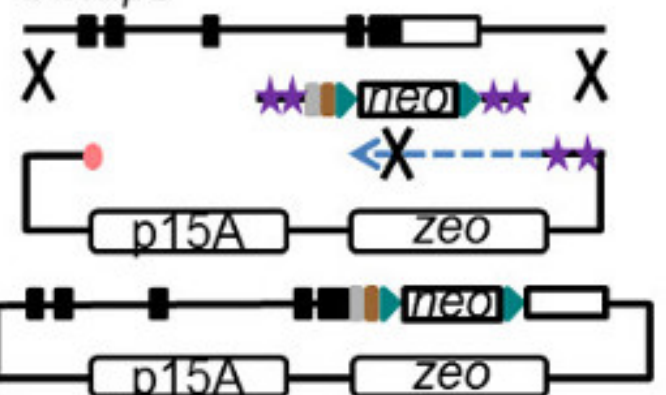

C

$\begin{array}{llllllllllllll}\mathrm{M} & 1 & 2 & 3 & 4 & 5 & 6 & 7 & 8 & 9 & 10 & 11 & 12 & \mathrm{C}\end{array}$

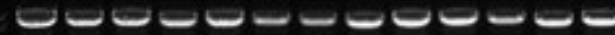

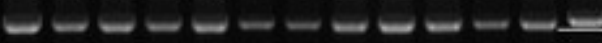

$---------\infty$

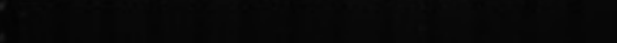

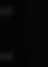

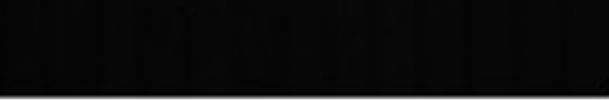

D

\section{P2rx1}
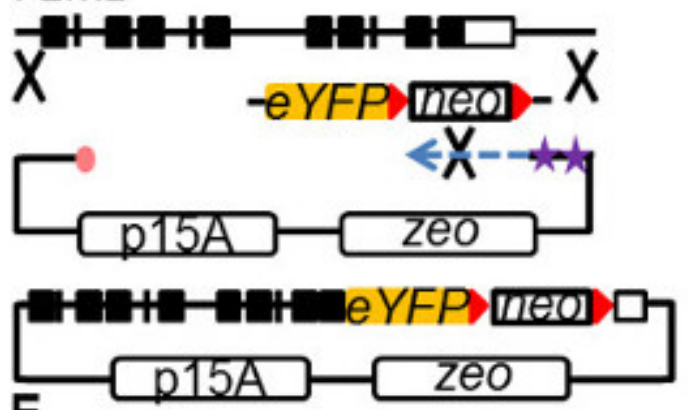

E

$\begin{array}{llllllllllllll}M & 1 & 2 & 3 & 4 & 5 & 6 & 7 & 8 & 9 & 10 & 11 & \mathrm{C} & \mathrm{M}\end{array}$

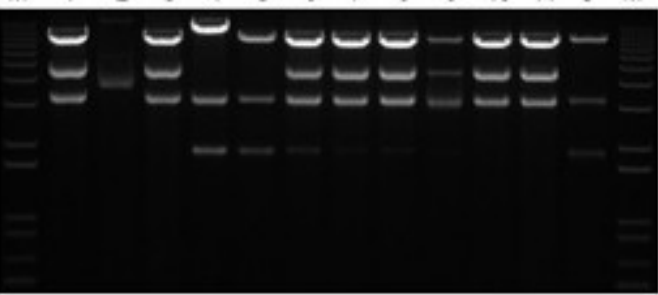

$\mathrm{kb}$

8.3

4.4
3.1

1.8

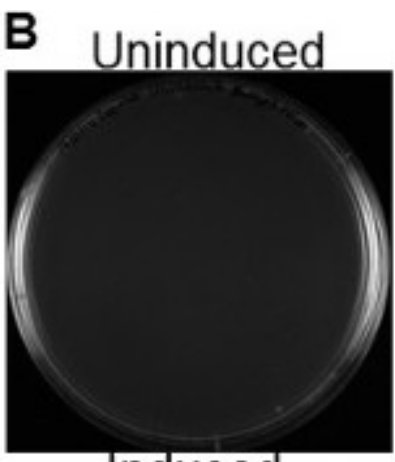

Induced

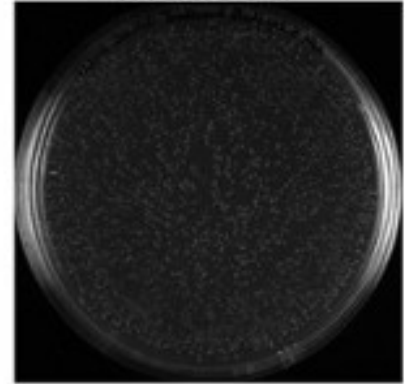

$2 X F L A G \unrhd C B P$

FRT

$\mathbf{F}$

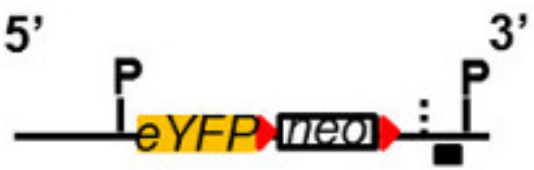

$\begin{array}{lllll}1 & 2 & 3 & 4 & 5\end{array}$

$11.5 \mathrm{kbeYFP}$ $8.9 \mathrm{~kb}$ WT

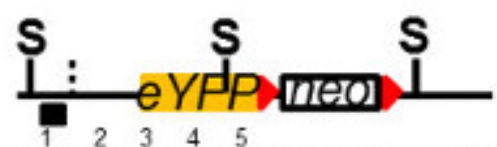

$7.6 \mathrm{~kb}$ eYFP

$6.9 \mathrm{~kb}$ WT

Figure 3. SPI enables construction of difficult knockin vectors. (A) Schematic of the SPI strategy used in the construction of the Dnttip1 dual tagged vector. Arrow indicates the direction of replication on the BAC clone. (B) Plating results of the uninduced and induced samples of the Dnttip1 SPI experiment. (C) EcoRI digest of Dnttip1 SPI clones. M, 1 kb ladder (NEB); C, p15A Dnttip1 gap repaired plasmid lacking the dual tag cassette. Fragment sizes are: tagged, $9.2+4.4+2.2 \mathrm{~kb}$; control; $9.2+4.6 \mathrm{~kb}$. (D) SPI based $P 2 r \times 1-e Y F P$ knockin vector construction. (E) EcoRI digest of $P 2 r x 1$-eYFP SPI clones. M, $1 \mathrm{~kb}+$ ladder (Invitrogen); C, p15A $P 2 r x 1$ gap repaired plasmid lacking the eYFP cassette. Fragment sizes are: tagged, $8.3+4.4+3.1+0.03 \mathrm{~kb}$; control; $8.3+3.1+1.8+0.03 \mathrm{~kb}$. (F) Southern blot analysis of $P 2 r \times 1-e Y F P$ gene targeting in JM8.N4 ES cell cells. Top panel, Southern blot with the 3' end probe using PshAl digest. Shown is the screening result of 5 clones. Bottom panel, Southern blot using the 5' end probe and Spel digest of the positives identified from the 3' end screening. PshAI RE site; S, Spel RE site. Dashed line represents the end of the vector HA. Black box denotes southern probe. Expected restriction fragments are, PshAl: WT, $8.9 \mathrm{~kb} ;$ eYFP-neo, 11.5 $\mathrm{kb}$; Spel: WT, $6.9 \mathrm{~kb}$; eYFP-neo, $7.6 \mathrm{~kb}$. Please click here to view a larger version of this figure. 
A

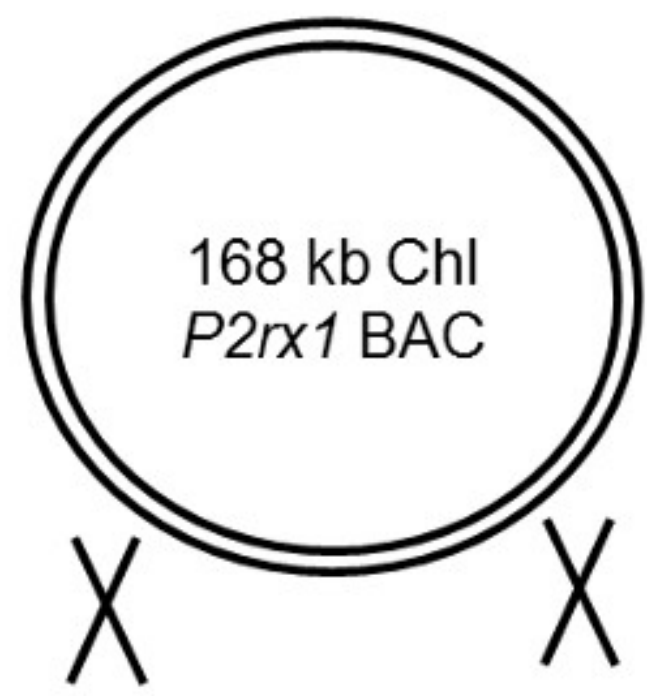

the YFP De]
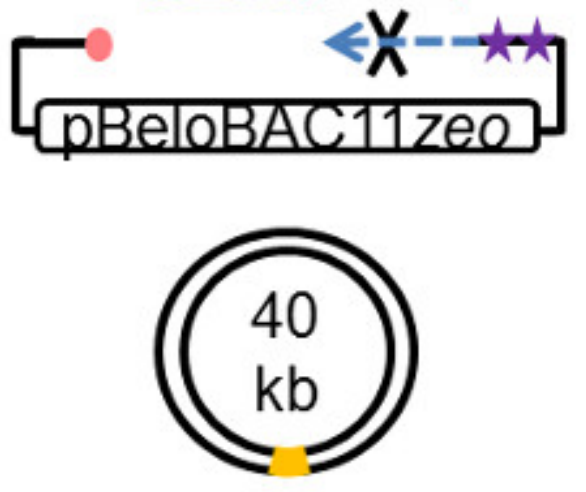

$\downarrow$

pBeloBAC11 zeo P2rx1 eYFP

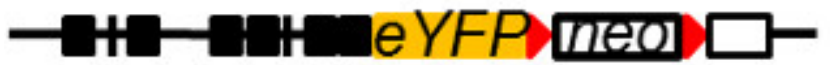

B

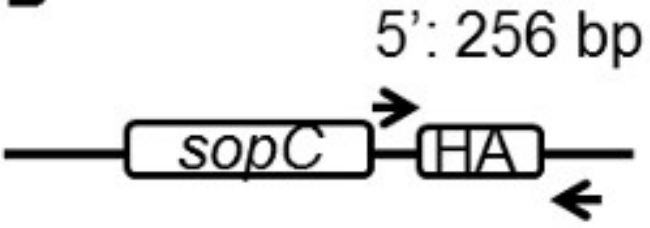

$\begin{array}{llllllll}M & 1 & 2 & 3 & 4 & 5 & 6 & P\end{array}$
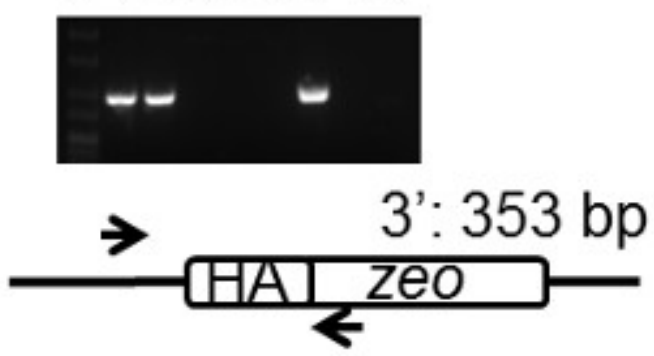

$\begin{array}{llllllll}M & 1 & 2 & 3 & 4 & 5 & 6 & P\end{array}$

$\sim-\sim \sim-\infty$
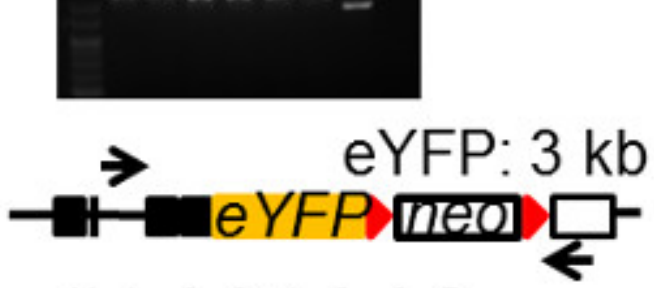

$\begin{array}{llllllll}M & 1 & 2 & 3 & 4 & 5 & 6 & \mathrm{P}\end{array}$

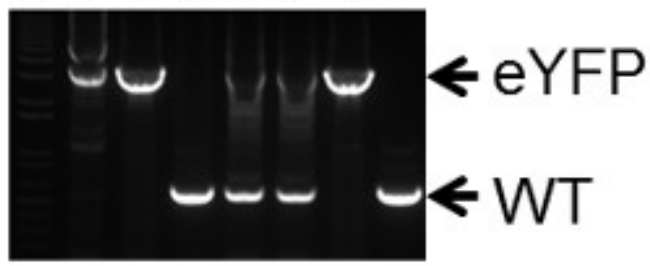

C

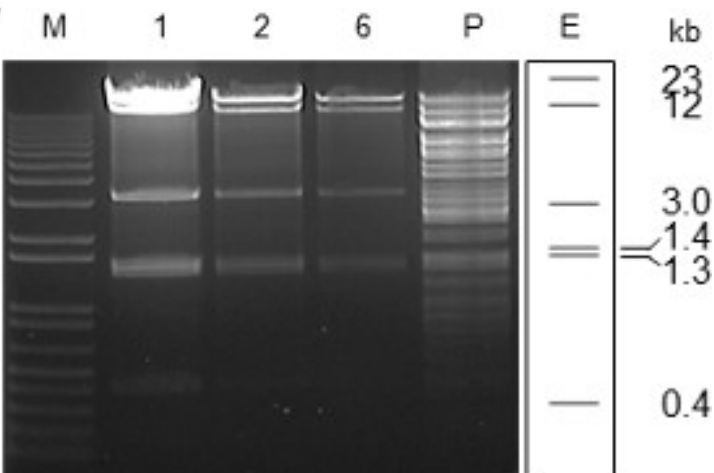

Figure 4. Simplified BAC trimming using SPI. (A) Schematic illustrating the concept of BAC trimming using SPI. Key to symbols is described in Figure 1. (B) PCR amplification across the 5' and 3' ends of the subcloned insert and across the P2rx1-eYFP insertion site. The screening strategy is shown for each type of PCR. M (top panel), Hyperladder $25 \mathrm{bp}$, (bottom panels), 1kb+; P, P2rx1 BAC; S, pBeloBAC11 zeo subcloning plasmid. (C) Hindlll digest of the three eYFP positive SPI BAC clones from (B); E, expected Hindlll restriction pattern of the trimmed eYFP BAC. Please click here to view a larger version of this figure. 
A

Zrsr2
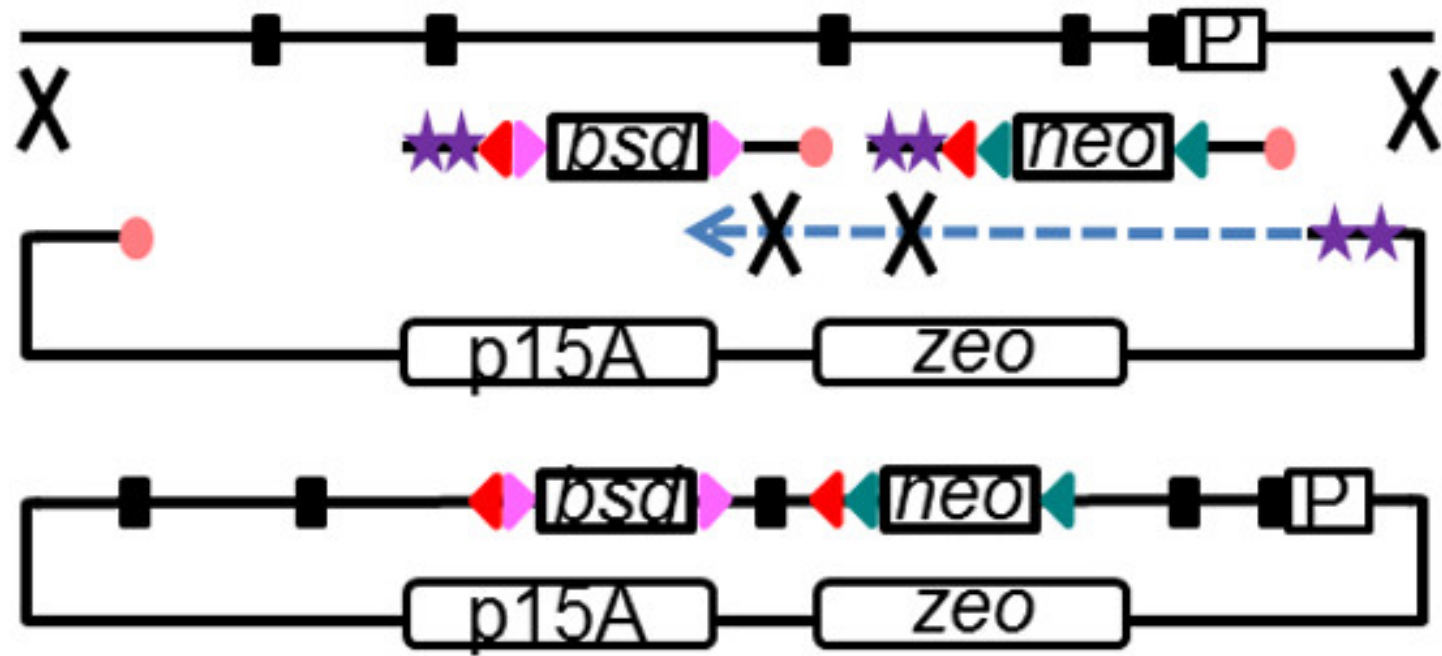

\section{Rox}

\section{ए Zrsr2 promoter}
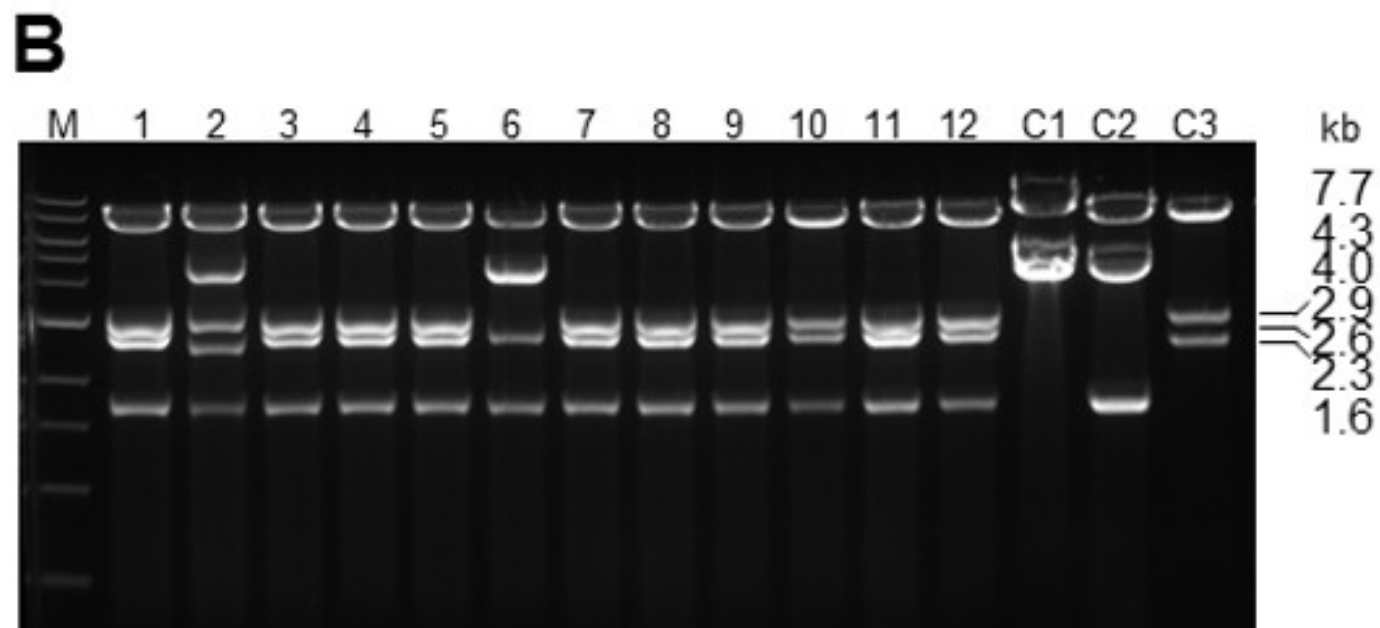

Figure 5. Conditional knockout vector generation using SPI cloning. (A) Schematic of the simultaneous insertion of two different LoxP flanked selection cassettes during subcloning of the Zrsr2 allele. Key to symbols is described in Figure 1. (B) EcoRI digests of Zrsr2 SPI clones. L, 1 kb ladder (NEB); C1, p15A ZrSr2 gap repaired plasmid, C2, p15A ZrSr2 gap repaired plasmid containing the neo insert; C3, p15A ZrSr2 gap repaired plasmid containing the $b s d$ insert. Fragment sizes are: $c k o, 7.7+2.9+2.6+1.6 \mathrm{~kb} ; \mathrm{C} 1,7.7+4.0 \mathrm{~kb} ; \mathrm{C} 2,7.7+4.3+1.6 \mathrm{~kb} ; \mathrm{C} 3,7.7+2.9+2.3$ $\mathrm{kb}$. Please click here to view a larger version of this figure.

\begin{tabular}{|l|l|l|}
\hline No of steps & Conventional recombineering pipeline ${ }^{\mathrm{a}}$ & Multiplex recombineering $^{\mathrm{b}}$ \\
\hline Step 1 & $\begin{array}{l}\text { Transformation of recombineering plasmid into } \\
\text { BAC host }\end{array}$ & $\begin{array}{l}\text { Transformation of recombineering plasmid } \\
\text { into BAC host. Preparation of the targeting } \\
\text { cassettes and subcloning vectors. }\end{array}$ \\
\hline Step 2 & Insertion of R1/R2 Gateway cassette & Multiplex gap repair cloning \\
\hline Step 3 & Insertion of floxed Kan cassette & O/N culture from single colonies \\
\hline Step 4 & Gap repair into R3/R4 plasmid & Plasmid preparation and verification \\
\hline Step 5 & Transformation into Cre+ E. coli & \\
\hline Step 6 & Plasmid preparation and verification & \\
\hline
\end{tabular}




\begin{tabular}{|c|c|c|}
\hline Step 7 & O/N three-way Gateway reaction & \\
\hline Step 8 & $\begin{array}{l}\text { Transformation of the three-way Gateway } \\
\text { reaction into } \mathrm{DH} 10 \mathrm{~B} \text { E. coli cells }\end{array}$ & \\
\hline Step 9 & Overnight culture from single colonies & \\
\hline Step 10 & Plasmid preparation and sequence verification & \\
\hline
\end{tabular}

Table 1: Comparison of conventional recombineering with SPI in the construction of conditional knockout vectors.

\begin{tabular}{|l|l|}
\hline RE buffer & $5 \mu \mathrm{l}$ \\
\hline DNA & $1 \mu \mathrm{g}$ plasmid or purified PCR products \\
\hline RE & $1 \mu \mathrm{l}(5$ units or more $)$ \\
\hline TE & up to $50 \mu \mathrm{l}$ \\
\hline Incubate at $37^{\circ} \mathrm{C}$ for at least $1 \mathrm{hr}$. Heat inacitvate according to the manufacturers instructions \\
\hline
\end{tabular}

\section{Table 2: RE digest.}

\begin{tabular}{|c|c|}
\hline PCR materials & Final concentration \\
\hline PCR buffer & $1 x$ \\
\hline dNTP & $200 \mathrm{nM}$ \\
\hline $\mathrm{MgSO}_{4}$ & $1.5 \mathrm{mM}$ \\
\hline Betaine & $1.3 \mathrm{M}$ \\
\hline DMSO & $1 \%$ \\
\hline Forward primer & $200 \mathrm{nM}$ \\
\hline Reverse primer & $200 \mathrm{nM}$ \\
\hline DNA polymerase & $1 U$ \\
\hline Template & $\begin{array}{l}10 \mathrm{ng} \text { of multicopy plasmids or } 2.5 \mu \text { of miniprep DNA for genotyping } \\
\text { PCRs }\end{array}$ \\
\hline Water & up to $50 \mu \mathrm{l}^{\mathrm{a}}$ \\
\hline \multicolumn{2}{|c|}{${ }^{\mathrm{a}}$ For a standard $50 \mu \mathrm{l} \mathrm{PCR}$ reaction with multicopy plasmids. Long range genotyping PCRs were set up in $25 \mu \mathrm{l}$ PCR reactions. } \\
\hline \multicolumn{2}{|l|}{ PCR conditions } \\
\hline $95^{\circ} \mathrm{C}$ & $2 \min$ \\
\hline $92^{\circ} \mathrm{C}$ & $10 \mathrm{sec}$ \\
\hline $55^{\circ} \mathrm{C}$ & $30 \mathrm{sec}$ \\
\hline $72^{\circ} \mathrm{C}$ & $30 \mathrm{sec}$ \\
\hline 30 cycles $^{b}$ & \\
\hline
\end{tabular}

\section{Table 3: PCR Set-up and Conditions.}

\begin{tabular}{|c|c|}
\hline Antibiotics & Concentration $^{\mathrm{a}}\left(\mu \mathrm{g} \mathrm{ml}^{-1}\right)$ \\
\hline Ampicllin & 50 \\
\hline Blasticidin $^{\mathrm{b}}$ & 40 \\
\hline Chloramphenicol & 12.5 \\
\hline Gentamicin & 2 \\
\hline Hygromycin $^{c}$ & 30 \\
\hline Kanamycin $^{\mathrm{b}}$ & 15 \\
\hline Tetracycline & 4 \\
\hline
\end{tabular}




\begin{tabular}{|l|l|}
\hline Trimethoprim $^{\mathrm{c}}$ & 10 \\
\hline Zeocin & 5 \\
\hline${ }^{\mathrm{a}}$ Recommended for use with BACs and multicopy plasmids when used in combinations in multiplex recombineering \\
\hline${ }^{\mathrm{b}}$ Blasticidin $\left(35 \mu \mathrm{g} \mathrm{ml}^{-1}\right)$ and Kanamycin $\left(6 \mu \mathrm{ml}^{-1}\right)$ when used together in combination \\
\hline${ }^{\mathrm{c}}$ Hygromycin and Trimethoprim are not recommended for selection with single copy BACs. \\
\hline
\end{tabular}

Table 4. Recommended antibiotic concentrations for use in SPI experiments.

\section{Discussion}

Construction of ES cell lines and mouse models has historically involved gene targeting using plasmid constructs that contained the modified allele $^{4}$. However, the construction of these complex gene targeting vectors has proved to be a significant bottleneck in the timely production of such models. The development of recombineering based vector construction strategies has allowed improved vector designs and more efficient vector assembly. Nonetheless, current recombineering protocols still involve multiple steps, require intermediate plasmid purification and use different bacterial strains. Subcloning plus insertion offers a novel approach to vector construction that can be performed in one electroporation event in the resident BAC host strain. The utility of SPI in gene targeting was tested here in a variety of vector construction applications. In all instances examined here, SPI proved to be efficient and the correct recombinant plasmid was produced. In the majority of the cases, the multiple different cassettes were correctly inserted in the targeting vector. Large DNA cassettes and vectors were easily accommodated in the SPI protocol and demonstrated the flexibility of this system.

SPI relies on the use of long homology sequences and phosphorothioate (PTO) protection of the linear DNA cassettes. PTO modification confers protection against exonucleases to the linear $D N A^{20,21}$ and the long $\mathrm{HA}$ increases recombination efficiency to permit multiplexing. However, synthesis of longer oligo sequences increases the chances of accumulating errors especially deletions. Mutations in the oligo can be particularly detrimental if they cover protein coding regions. DNA sequencing across the HA and covering the full-length of the inserted cassette is highly recommended to eliminate clones with any sequence alterations. Use of a high-fidelity DNA polymerase system is also suggested to avoid introduction of any PCR errors. The length and composition of the HA of the subcloning plasmid is more critical relative to that of the insertion cassette (data not shown). For particularly sensitive applications like construction of knockin vectors, where any mutations in exon regions are not tolerated, the HA of the insertion cassette can be shortened (50-120 bp) to avoid problems associated with long oligos. The insertion cassette can also be left unmodified or dual phosphorothioated (where knowledge of the direction of replication is not available). But multiplexing in these cases still requires long protected HA subcloning plasmids. A caveat of this particular strategy is the lowering of multiplexing efficiency that can potentially impact SPI cloning at different loci.

The length of the insertion cassette and the subcloning plasmid is another important parameter in SPI cloning. Larger DNA molecules electroporate less efficiently and the effect is cumulative, given the requirement to introduce all the cassettes in the same cell (data not shown). Multiplexing is most efficient with smaller insertion cassettes. DNA fragments larger than $3 \mathrm{~kb}$ also place a limit on Red mediated ssDNA processing, which is most efficient up to $3 \mathrm{~kb}^{30}$. Insertion cassettes exceeding $3 \mathrm{~kb}$ are dual resected and recombine less efficiently via a beta independent pathway ${ }^{20}$. Therefore, screening of sufficient colonies to identify the correct clone becomes important in these cases. A longer duration of recombination post electroporation also increases the chances of recovery of the correct clone in difficult SPI exercises. Up to four small cassettes $(<1.5 \mathrm{~kb})$ can be inserted simultaneously with the SPI process, though the multiplexing efficiency decreases with each additional cassette (data not shown). However, this reflects the outcome of an optimal SPI experiment and it is advisable to consider the limits of multiplexing when using many large cassettes.

The development of genome editing tools like clustered regularly interspaced short palindromic repeats (CRISPR)-cas9 endonuclease system has enabled the creation of novel genome modifications and has led to more efficient genome engineering ${ }^{36}$. However, these newer technologies have complemented gene targeting vectors rather than supplanted them. It is envisaged that the CRISPR-cas system could replace antibiotic selection and further refine the multiplex recombineering protocol.

\section{Disclosures}

T.R.R is the inventor of a British patent application on multiplex recombineering technology.

\section{Acknowledgements}

T.R.R conceived the project, designed and performed experiments and prepared the manuscript. E.J.K and S.E.M performed experiments. All authors contributed intellectually to the final manuscript. This work was funded by a University of Leicester Innovation Fellowship to SEM and by a UK Medical Research Council (MRC) Senior Research Fellowship to S.M.C (MR/J009202/1). DNA sequencing was performed by Protein and Nucleic Acid Laboratories (PNACL), Centre for Core Biotechnology Services, University of Leicester. We wish to thank A. Francis Stewart for providing the Rox and Dre plasmids. pL451 and pL452 plasmids were obtained from National Cancer Institute (NCl), Frederick, USA.

\section{References}

1. Dovey, O. M., Foster, C. T., \& Cowley, S. M. Histone deacetylase 1 (HDAC1), but not HDAC2, controls embryonic stem cell differentiation. Proc. Natl. Acad. Sci. USA. 107, 8242-8247, doi:10.1073/pnas.1000478107 (2010). 
2. Harvey, M. et al. Spontaneous and carcinogen-induced tumorigenesis in p53-deficient mice. Nat. Genet. 5, 225-229, doi:10.1038/ng1193-225 (1993).

3. Waterhouse, P. et al. Lymphoproliferative disorders with early lethality in mice deficient in Ctla-4. Science. 270, 985-988, doi:10.1126/ science.270.5238.985 (1995).

4. Thomas, K. R., \& Capecchi, M. R. Site-directed mutagenesis by gene targeting in mouse embryo-derived stem cells. Cell. 51, 503-512 (1987).

5. Hasty, P., Rivera-Pérez, J., \& Bradley, A. The length of homology required for gene targeting in embryonic stem cells. Mol. Cell. Biol. 11, 5586-5591, doi:10.1128/mcb.11.11.5586 (1991).

6. Smithies, O., Gregg, R. G., Boggs, S. S., Koralewski, M. A., \& Kucherlapati, R. S. Insertion of DNA sequences into the human chromosomal beta-globin locus by homologous recombination. Nature. 317, 230-234 (1985).

7. Hirata, R., Chamberlain, J., Dong, R., \& Russell, D. W. Targeted transgene insertion into human chromosomes by adeno-associated virus vectors. Nat. Biotechnol. 20, 735-738 (2002).

8. Szostak, J. W., Orr-Weaver, T. L., Rothstein, R. J., \& Stahl, F. W. The double-strand-break repair model for recombination. Cell. 33, 25-35 (1983).

9. Bradley, A., Evans, M., Kaufman, M. H., \& Robertson, E. Formation of germ-line chimaeras from embryo-derived teratocarcinoma cell lines. Nature. 309, 255-256 (1984).

10. Fu, J., Teucher, M., Anastassiadis, K., Skarnes, W., \& Stewart, A. F. A recombineering pipeline to make conditional targeting constructs. in: Methods in Enzymology. Volume 477, Wassarman, P.M.., \& Soriano P. M., eds., Academic Press, 125-144, (2010).

11. Zhang, Y., Buchholz, F., Muyrers, J., \& Stewart, A. A new logic for DNA engineering using recombination in Escherichia coli. Nat. Genet. 20 , $123-128$ (1998).

12. Copeland, N., Jenkins, N., \& Court, D. Recombineering: a powerful new tool for mouse functional genomics. Nat. Rev. Genet. 2, 769 - 779 (2001).

13. Muyrers, J., Zhang, Y., Testa, G., \& Stewart, A. Rapid modification of bacterial artificial chromosomes by ET-recombination. Nucleic Acids Res. 27, 1555 - 1557 (1999).

14. Yu, D. et al. An efficient recombination system for chromosome engineering in Escherichia coli. Proc. Natl. Acad. Sci. USA. 97,5978 - 5983 (2000).

15. Sharan, S. K., Thomason, L. C., Kuznetsov, S. G., \& Court, D. L. Recombineering: a homologous recombination-based method of genetic engineering. Nature Protoc. 4, 206-223, doi:10.1038/nprot.2008.227 (2009).

16. Wang, J. et al. An improved recombineering approach by adding RecA to $\lambda$ red recombination. Mol. Biotechnol. 32, 43-53 (2006).

17. Datta, S., Costantino, N., \& Court, D. L. A set of recombineering plasmids for gram-negative bacteria. Gene. 379, 109-115, doi:10.1016/ j.gene.2006.04.018 (2006).

18. Fu, J. et al. Full-length RecE enhances linear-linear homologous recombination and facilitates direct cloning for bioprospecting. Nat. Biotechnol. 30, 440-446 (2012).

19. Muyrers, J., Zhang, Y., Buchholz, F., \& Stewart, A. RecE/RecT and Redalpha/Redbeta initiate double-stranded break repair by specifically interacting with their respective partners. Genes Develop. 14, 1971 - 1982 (2000).

20. Maresca, M. et al. Single-stranded heteroduplex intermediates in lambda Red homologous recombination. BMC Mol. Biol. 11, 54 (2010).

21. Mosberg, J. A., Lajoie, M. J., \& Church, G. M. Lambda Red recombineering in Escherichia coli occurs through a fully single-stranded intermediate. Genetics. 186, 791-799, doi:10.1534/genetics.110.120782 (2010).

22. Liu, P., Jenkins, N. A., \& Copeland, N. G. A highly efficient recombineering-based method for generating conditional knockout mutations. Genome Res. 13, 476-484, doi:10.1101/gr.749203 (2003).

23. Chan, W. et al. A recombineering based approach for high-throughput conditional knockout targeting vector construction. Nucleic Acids Res. 35, e64, doi:10.1093/nar/gkm163 (2007).

24. Zhang, Y., Muyrers, J. P. P., Testa, G., \& Stewart, A. F. DNA cloning by homologous recombination in Escherichia coli. Nat. Biotechnol. 18, 1314-1317 (2000).

25. Lee, E. C. et al. A highly efficient Escherichia coli-based chromosome engineering system adapted for recombinogenic targeting and subcloning of BAC DNA. Genomics. 73, 56-65, doi:10.1006/geno.2000.6451 (2001).

26. Skarnes, W. C. et al. A conditional knockout resource for the genome-wide study of mouse gene function. Nature. 474, 337-342 (2011).

27. Valenzuela, D. M. et al. High-throughput engineering of the mouse genome coupled with high-resolution expression analysis. Nat. Biotechnol. 21, 652-659 (2003).

28. Bantscheff, M. et al. Chemoproteomics profiling of HDAC inhibitors reveals selective targeting of HDAC complexes. Nat. Biotechnol. 29, 255-265, doi:10.1038/nbt.1759 (2011).

29. Mulryan, K. et al. Reduced vas deferens contraction and male infertility in mice lacking P2X1 receptors. Nature. 403, 86-89, doi:10.1038/47495 (2000).

30. Subramanian, K., Rutvisuttinunt, W., Scott, W., \& Myers, R. The enzymatic basis of processivity in lambda exonuclease. Nucleic Acids Res. 31, 1585 - 1596 (2003).

31. Antoch, M. P. et al. Functional identification of the mouse circadian Clock gene by transgenic BAC rescue. Cell. 89, 655-667 (1997).

32. Rostovskaya, M. et al. Transposon-mediated BAC transgenesis in human ES cells. Nucleic Acids Res. 40, e150, doi:10.1093/nar/gks643 (2012).

33. Giraldo, P., \& Montoliu, L. Size matters: use of YACs, BACs and PACs in transgenic animals. Transgenic Res. 10, 83-103 (2001).

34. Hill, F. et al. BAC trimming: minimizing clone overlaps. Genomics. 64, 111-113, doi:10.1006/geno.1999.6106 (2000).

35. Warming, S., Costantino, N., Court, D. L., Jenkins, N. A., \& Copeland, N. G. Simple and highly efficient BAC recombineering using galK selection. Nucleic Acids Res. 33, e36, doi:10.1093/nar/gni035 (2005).

36. Cong, L. et al. Multiplex Genome Engineering Using CRISPR/Cas Systems. Science. 339, 819-823, doi:10.1126/science.1231143 (2013). 